\title{
The relationship between the Leicester cough questionnaire, eosinophilic airway inflammation and asthma patient related outcomes in severe adult asthma
}

\author{
Sushiladevi Natarajan', Robert C. Free', Peter Bradding ${ }^{1}$, Lorcan McGarvey² and Salman Siddiqui ${ }^{\text {** }}$
}

\begin{abstract}
Background: Severe asthma is characterised by a variety of symptoms, which include chronic cough, however the mechanisms responsible for cough reflex hypersensitivity in asthma remain poorly elucidated. Current asthma patient-related outcome instruments such as the six-point Juniper Asthma Control Score (ACQ-6) and Asthma Quality of Life Questionnaire (AQLQ) were not primarily designed to capture cough and its related morbidity in asthma. The Leicester Cough Questionnaire (LCQ) is a patient-related outcome instrument designed to capture the health-related quality of life associated with cough. To date the LCQ has not been evaluated in a severe asthma population.

Methods: We evaluated 262 extensively characterised adult patients with severe asthma attending the Leicester Severe Asthma Service. All patients had a clinician diagnosis of asthma and objective physiological evidence and met the ATS/ERS criterion for servere asthma. In all patients we evaluated a) the LCQ distribution and b) the relationships between the LCQ and ACQ-6, AQLQ, airway inflammation in sputum.

Results: The LCQ demonstrated the following properties; mean: 15.0, standard deviation: 4.54, median: 15.48, and range: 11.6-19.2. We found a moderate correlation between LCQ and ACQ-6 $(r=-0.605, p<0.0001)$ and a LCQ and AQLQ $(r=0.710, p<0.0001)$. There was no relationship between LCQ and $\log _{10}$ sputum percentage eosinophils (\%).

Conclusion: A proportion of patients with severe asthma have a significant degree of cough-related morbidity that appears independent of eosinophilic airway inflammation and is not captured fully by existing asthma patient-reported outcome instruments. Our preliminary findings suggest that further research is now required to validate the LCQ and its responsiveness in severe asthma populations to capture cough-related morbidity and response to specific interventions.
\end{abstract}

Keywords: Asthma, Cough

\section{Introduction}

Severe asthma is characterised by suboptimal asthma control in patients receiving step $4 / 5$ therapy [1] and is associated with high levels of health care utilisation [2]. Severe asthma is associated with a range of co morbidities

\footnotetext{
* Correspondence: ss338@le.ac.uk

'Department of Infection, Immunity and Inflammation, NIHR Respiratory Biomedical Research Unit, Glenfield Hospital, University of Leicester, Leicester, UK

Full list of author information is available at the end of the article
}

[3] (such as nasal polyps, suboptimal treatment adherence, as well as treatment refractory and difficult asthma) [1, 4].

Chronic cough is associated with physical and psychological morbidity [5], however the prevalence of chronic cough within severe asthma populations has not been determined, in part due to the lack of well-validated assessment tools.

Cough reflex hypersensitivity syndrome [6] may underlie the aetiology of chronic cough but the mechanisms are poorly understood and therapeutic options are limited. Similarly, the impact of chronic cough on the 
health status of patients with severe asthma is not well understood as existing asthma patient-related outcome measures such as the six-point Juniper Asthma Control Questionnaire (ACQ-6) and Asthma Quality of Life Questionnaire (AQLQ) were not primarily designed to capture cough and its related morbidity. There are a number of tools that can be used to understand and phenotype cough which include digital cough monitors [7], cough specific health status questionnaires such as the Leicester Cough Questionnaire (LCQ) and cough visual analogue scales $[8,9]$. The LCQ is a patientreported questionnaire designed to capture the healthrelated quality of life of cough and is responsive to changes in chronic cough [10]. More recently the LCQ has been used in asthma and chronic obstructive pulmonary disease (COPD) patients to assess the impact of gastro-oesophageal reflux disease (GORD) on coughrelated morbidity [11] and has also been validated against the SF-12 questionnaire in COPD [12]. However the LCQ has not been evaluated in large carefully characterised severe asthma populations to date.

We sought to determine the spectrum of cough related morbidity in a severe asthma population, by measuring total LCQ and its sub domains and evaluating its relationship with standard asthma related questionnaires (ACQ-6, AQLQ) and percentage sputum eosinophils (a marker of the degree of eosinophilc bronchitis -a reported driver of cough in asthma).

\section{Methods}

We evaluated 312 adult patients referred to the Leicester Difficult Asthma Service, using data from a local clinical management database. The database included all referral from primary and secondary care.

The use of data extracted from the local database for the purposes of research was approved by the Leicestershire, Northamptonshire, \& Rutland Research Ethics Committee (REC Reference.13/EM/0287) and all patients gave their written informed consent.

The Leicester difficult asthma service, is a tertiary referral service for difficult asthma patients in the UK and receives both local and national referrals to evaluate patients with difficult to control asthma. The evaluation includes (i) confirmation of the diagnosis of asthma in all patients using objective measurements, including extensive phenotyping assessments (ii) systematic evaluation and treatment of comorbidities e.g., rhinosinusitis, non-adherence and (iii) evaluation for biological agents and bronchial thermoplasty after (i) and (ii).

All patients $(n=312)$ had an expert clinician diagnosis of asthma and one or more of the following objective physiological criteria; (i) positive methacholine $\mathrm{PC}_{20} \leq$ $8 \mathrm{mg} / \mathrm{ml}$, (ii) bronchodilator responsiveness of $200 \mathrm{ml}$ and $12 \%$ following the administration of $200 \mu \mathrm{g}$ of inhaled salbutamol or (iii) peak flow variation over a two week period of $\geq 20 \%$ [1].

$22 / 312$ patients were excluded from analyses as they did not have difficult to treat asthma and had milder disease (GINA treatment steps 1-3).

262/290 patients met the criteria met the American Thoracic Society/European Respiratory Society consensus criteria for severe asthma [13]. Therefore our final population for analysis consisted entirely of patients with severe asthma, defined according to international guidelines, all of whom had objective evidence of asthma and were being assessed and managed in a tertiary severe asthma centre in the UK.

Phenotyping assessments were performed in the stable state and in patients that had been free from exacerbations for $\geq 6$ weeks. Patients underwent lung function measurements, including spirometry [post bronchodilator $(400 \mu \mathrm{g}$ salbutamol via a metered dose inhaler and spacer)], induced sputum samples for differential cell count and completed the LCQ, ACQ-6 and AQLQ questionnaires at a single clinical visit. Due to the association of cough with reflux and upper airway disease, selfreported gastrointestinal reflux, defined as the presence of heartburn, cough, nausea, chest pain or dysphagia after meals was documented, whilst the presence or absence of seasonal/perennial rhinitis and nasal polyposis was also recorded.

The LCQ is a nineteen-point questionnaire capturing three domains; Physical (8 items), Psychological (7 items) and Social (4 items). Each item has a score range 1 to 7 . Item scores for each domain are summed and divided by the total number of items in each domain. The total LCQ is the sum of all domain scores. The minimum and maximum achievable LCQ total scores are 3 and 21 respectively [10]. A lower LCQ score signifies more cough. The LCQ Minimal clinically important difference (MCID) is 1.3 in chronic cough [14]. The Global Lung function Initiative (GLI) reference equations were used to calculate $\mathrm{FEV}_{1} \mathrm{Z}$ score. Patients with abnormal spirometry had an $\mathrm{FEV}_{1}$ standardised residual of $\leq-1.64$.

\section{Statistical analysis}

Statistical analysis was performed using SPSS 22 (IBM Corporation, Somers, NY, USA) and Prism 6 (GraphPad Software Inc., La Jolla, California, USA). Data were analysed using Student's $t$-test or Mann-Whitney $U$ test for parametric and non-parametric data respectively and chi squared test for proportions. The threshold for statistical significance was set at $p<0.05$. Correlations between continuous variables were calculated using Pearson's correlation coefficient. 


\section{Results}

The clinical characteristics of the asthma population are shown in Table 1. The population reported here was similar to other UK severe asthma populations [15]. We did not systematically exclude smokers (see Table 1), however only $3 / 42$ patients with a smoking history $\geq 10$ pack years had spirometrically confirmed fixed airflow obstruction (compatible with ACOS).

Figure 1a-d, shows the LCQ total and domain distributions. The LCQ demonstrated the following properties: a) Mean: 15.0, b) Standard deviation: 4.54, c) Median: 15.48 , c) IQR: $11.6-19.2$, d) Range: $4.0-21$, e) $10^{\text {th }}$ percentile point: 8.20 and f) $90^{\text {th }}$ percentile point: 20.38 .

There was a moderate negative correlation between LCQ and ACQ-6 $(r=-0.605, p<0.0001)$ (see Fig. $2 b)$, and a moderate positive correlation between LCQ and AQLQ $(r=0.710, p<0.0001)$ (Fig. 2a). There was no correlation between LCQ and $\log _{10}$ sputum eosinophils (\%) $(r=-0.106, p=0.106)$ or sputum neutrophils (data not shown). Similar and statistically significant correlations existed between LCQ and ACQ-6/AQLQ in GINA 4 and GINA 5 severe asthma populations respectively (data not shown).

The female asthma population (62.6\%) had an LCQ of 14.9 (10.8-18.7), significantly lower LCQ when compared to males 17.1 (12.8-19.8), $p=0.0055$.

Patients with asthma with self-reported gastrointestinal reflux $(47.3 \%)$ were more likely to be female $(67.7 \%$, $p<0.0001)$ and had a significantly lower LCQ compared to patients without self-reported gastrointestinal reflux [median (IQR): 14.3 (10.8-17.8) vs.16.5 (12.819.7), $p=0.0015]$. However there was no significant difference in LCQ between female patients with asthma with self-reported gastrointestinal reflux (32.0\%) and without self-reported gastrointestinal reflux (30.5\%) [median (IQR): 14.31 (10.9-16.9) vs. 15.83 (10.2-19.5), $p=0.1835]$.

There was no significant difference in LCQ between patients with asthma with nasal polyps and patients with asthma without nasal polyps [median (IQR): 14.7 (10.7$19.7)$ vs. 15.5 (11.9-18.8), $p=0.7906]$. There was also no significant difference in LCQ between patients with asthma with perennial/seasonal rhinitis compared to patients with asthma without perennial/seasonal rhinitis [median (IQR): 15.21 (11.6-19.0) vs. 16.36 (15.7-20.0), $p=0.3680$ ).

There was no significant difference in the total LCQ score between smokers with $\geq 10$ pack/years and smokers with $<10$ pack/years (median (IQR): 15.4 (12.7$19.8)$ vs.16.22 (11.3-19.0), $p=0.5755)$.

\section{Discussion}

We have chosen the $\mathrm{LCQ}$, a simple and objective measure of cough to evaluate the burden of cough in severe asthma
Table 1 Clinical Characteristics of Severe Asthma Population

\begin{tabular}{|c|c|}
\hline & $\begin{array}{l}\text { Asthma population } \\
(n=262)\end{array}$ \\
\hline Age (years) & $54.03(14.9)$ \\
\hline Sex (\% male) & 37.4 \\
\hline GINA Treatment Step ${ }^{a}$ & $\begin{array}{l}5(4-5) \\
\text { Step 4: } 124 \\
\text { Step 5: } 96\end{array}$ \\
\hline Sputum Eosinophil (\%) ${ }^{a}$ & $3.5(0.5-18.75)$ \\
\hline$\%$ on ICS/LABA combination therapy (number) & $94.5(n=247)$ \\
\hline$\%$ on Montelukast (number) & $27.1(n=71)$ \\
\hline$\%$ on oral theophylline (number) & $32.4(n=85)$ \\
\hline$\%$ on Inhaled Tiotrpoium Bromide (number) & $26.0(n=68)$ \\
\hline$\%$ on maintenance oral prednisolone (number) & $53.8(n=156)$ \\
\hline Dose $(\mathrm{mg} / 24 \mathrm{~h})$ in those on Prednisolone & $10(5.0-12.5)^{\mathrm{a}}$ \\
\hline Self-reported gastrointestinal reflux (\%) & 49.0 \\
\hline Nasal polyps (\%) (number) & $26.3(n=69)$ \\
\hline Perennial/seasonal rhinitis \% (number) & $58.3(n=153)$ \\
\hline Smoking history (pack years) & $7.2(2.6-20)$ \\
\hline Exacerbations in past year & $3(1.0-6.0)^{\mathrm{a}}$ \\
\hline ITU admissions in past year & $0.0(0.0-0.0)^{\mathrm{a}}$ \\
\hline Total ITU admissions & $0.0(0.0-1.0)^{\mathrm{a}}$ \\
\hline Hospital admissions in past year & $0.0(0.0-1.0)^{\mathrm{a}}$ \\
\hline Unscheduled GP/A\&E visits & $3.0(0.0-7.0)^{\mathrm{a}}$ \\
\hline Smoking history (pack years) & $7.2(2.6-20)^{\mathrm{a}}$ \\
\hline Proportion of current: ex smokers & $\begin{array}{l}\text { Current smokers: } n=19 \\
\text { Ex-smokers: } n=76\end{array}$ \\
\hline \% Smoking >10 pack years (number) & $16.0(n=42)$ \\
\hline Post Bronchodilator FEV $1 \%$ Predicted) & $73.33(35.7)$ \\
\hline Post Bronchodilator FVC (\% Predicted) & $96.6(154.4)$ \\
\hline Post Bronchodilator $\mathrm{FEV}_{1} / \mathrm{FVC}(\%)$ & $66.82(14.6)$ \\
\hline GLI FEV 1 Z score & $-1.67(2.68)$ \\
\hline Bronchodilator reversibility (\%) & $14.13(19.01)$ \\
\hline ACQ-6 score ${ }^{a}$ & $2.50(1.5-3.5)^{\mathrm{a}}$ \\
\hline AQLQ score ${ }^{a}$ & $3.83(3.0-5.1)^{\mathrm{a}}$ \\
\hline LCQ Total score ${ }^{a}$ & $15.48(11.6-19.2)^{a}$ \\
\hline LCQ Total score (females) ${ }^{a}$ & $14.9(10.8-18.7)[n=164]$ \\
\hline LCQ Total Score (males) ${ }^{\mathrm{a}}$ & $17.1(12.8-19.8)[n=98]$ \\
\hline LCQ Total score (smoker <10 pack/years) & $16.22(11.3-19.0)$ \\
\hline LCQ Total score (smoker $\geq 10$ pack/years) & $15.4(12.7-19.8)$ \\
\hline LCQ Physical ${ }^{\mathrm{a}}$ & $4.75(3.6-6.0)^{\mathrm{a}}$ \\
\hline LCQ Psychological ${ }^{\mathrm{a}}$ & $5.57(4.0-6.7)^{\mathrm{a}}$ \\
\hline LCQ Social $\left.\right|^{a}$ & $5.38(3.8-6.8)^{\mathrm{a}}$ \\
\hline
\end{tabular}

GINA Global Initiative for Asthma, ICS/LABA inhaled corticosteroids/long acting beta-2 agonist combination therapy, ITU Intensive care unit, GP General practice, A\&E Accident and emergency, $F E V_{1}$ Forced Expiratory Volume in one second, $F V C$ Forced Vital Capacity, GLI Global Lung Function Initiative, ACQ-6 Six-point Asthma Control Questionnaire, AQLQ Asthma Quality of Life Questionnaire, LCQ Leicester Cough Questionnaire. Data expressed as mean (standard deviation) or ${ }^{\mathrm{a}}$ median (interquartile range) 

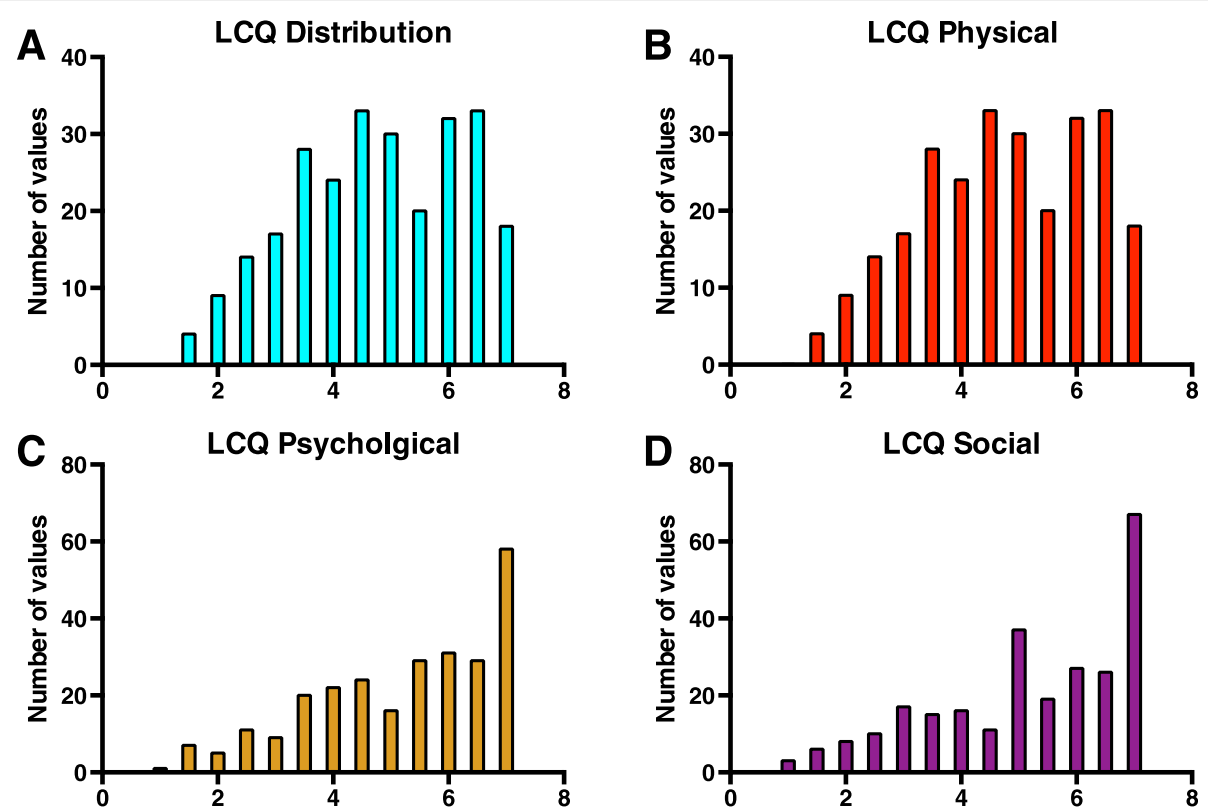

Fig. 1 Distribution of the Leicester Cough Questionnaire. $L C Q=$ Leicester Cough Questionnaire. Panel a: Histogram of the distribution of the Leicester cough questionnaire, Panel b-d: Histogram of the distribution of Leicester cough questionnaire domains

and its relationships to existing asthma patient-related outcome measures and eosinophilic airway inflammations.

We identified that a significant proportion of patients with severe asthma demonstrated high levels of coughrelated morbidity as evidenced by the LCQ. The $10^{\text {th }}$ percentile LCQ (8.20) and median LCQ (15.48) in severe asthma when compared to other chronic respiratory conditions including Idiopathic chronic cough [median (SD): 12.8 (3.7)] [16] and Idiopathic pulmonary fibrosis (IPF) [mean (SD): 16.16 (3.66)] [17] indicated a similar degree of cough related morbidity. Although a previous study reported a median LCQ (range): 20.2 (9.5-1.0) [11], this particular study assessed patients with varying degrees of asthma severity, suggesting that our observations are likely to be confined to patients with severe asthma.

The LCQ was initially developed to study idiopathic chronic cough [10]. Existing validated asthma control and quality of life instruments either have no coughrelated questions (e.g., ACQ) or limited information on

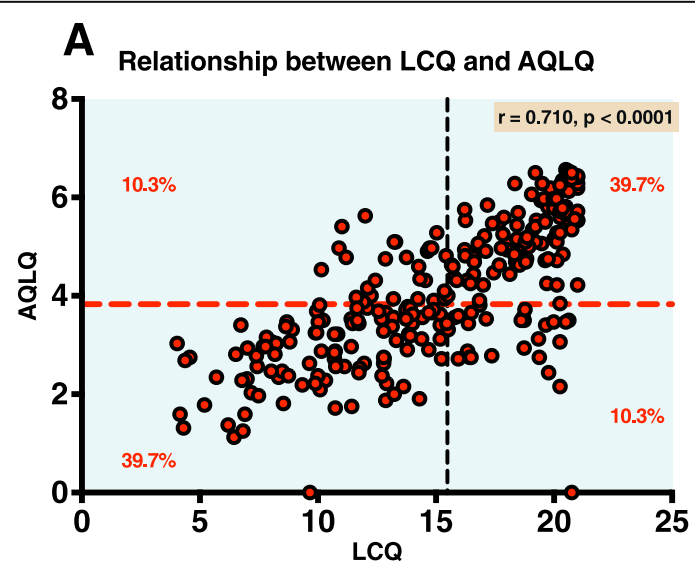

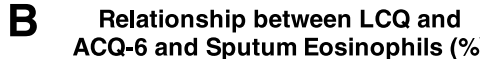

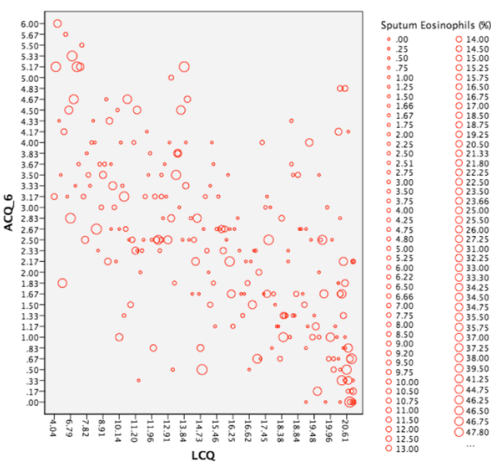

Fig. 2 The relationships between the Leicester Cough Questionnaire and Eosinophilic airway Inflammation in sputum and Asthma Patient-Related Outcomes in Severe Asthma. LCQ = Leicester Cough Questionnaire, ACQ-6 = Six-point Asthma Control Questionnaire, AQLQ = Asthma Quality of Life Questionnaire Panel a: Correlation between the Leicester cough questionnaire and Asthma quality of life questionnaire. Pearson correlation coefficient: $r=0.710, p<0.0001$. Dashed line represents median. Percentage of subjects in each quadrant is shown. Panel $\mathbf{b}$ : Bubble plot representing correlations between the Leicester cough questionnaire and sputum eosinophils (\%) (Pearson correlation coefficient: $r=-0.106, p=0.106)$ and Six-point Asthma control questionnaire (Pearson correlation coefficient: $r=.0 .605, p<0.0001$ 
cough related morbidity (e.g., AQLQ -has only one question cough-related question). We identified only modest correlations between LCQ and ACQ, AQLQ, which may suggest that the degree of cough is either cause or consequence of poor disease control and impaired health related quality of life. However further studies evaluating interventions that are known to modify asthma control and quality of life would be required to test whether chronic cough has a causal impact upon asthma control and quality of life in patients with severe asthma. Our observations support the role of LCQ as an additional phenotyping tool in patients with severe asthma.

Gastrointestinal reflux is a common cause of chronic cough and can coincide with asthma, rendering it a potential confounder of cough in asthma. Patients with asthma with self-reported gastrointestinal reflux were predominately female with a significantly lower LCQ compared to patients without self-reported gastrointestinal reflux. In support of this observation previous studies have shown subjects with asthma and concurrent GORD, had poorer asthma control and impaired cough -related quality of life [11]. However, there was no significant difference in LCQ between female patients with asthma, with and without self-reported gastrointestinal reflux. These observations suggest a heightened cough reflex sensitivity that is independent of reflux in female patients with severe asthma which has been well reported previously in female patients with idiopathic chronic cough [18].

One limitation of our study is that we did not quantify gastrointestinal reflux using objective measurements, which is important as reflux is well recognised to be silent in some patients. We also quantified upper airway disease due to rhinitis and nasal polyps using selfreporting, however in our experience self-reported disease demonstrates a high concordance with nasendoscopic findings, suggesting that our observations demonstrating a lack of association of LCQ with upper airways disease are valid.

Cough is also a well-recognised symptom in bronchiectasis and it is important to note that qualitative CT has demonstrated evidence of subclinical bronchiectasis in a proportion of patients (approximately 30\%) with severe asthma [19]. Therefore the presence of super added bronchiectasis may have been a potential contributor to cough in this population.

It is believed that chronic cough in asthma may be related to an eosinophilic bronchitis [20]. Airway eosinophils are associated with cough in the presence and absence of airway hyperresponsiveness [20] and mechanistically eosinophils may interact with muscarinic nerves [21] and promote cough reflex hypersensitivity. However, we found no significant relationship between sputum eosinophils (\%) and LCQ.
Recent cough guidelines [6] suggest cough reflex hypersensitivity syndrome underlies the aetiology of chronic cough; arising from the hypersensitivity of airway sensory nerves, and thus may be an underlying mechanism attributing to cough in severe asthma. Pharmacological therapies targeting cough reflex hypersensitivity include P2X3 receptor antagonists [22].

\section{Conclusions}

We conclude that a significant proportion of patients with severe asthma have a high degree of cough-related morbidity that appears independent of eosinophilic airway inflammation, is associated with suboptimal disease control and quality of life. Our preliminary findings suggest that further research is now required to validate the LCQ and its responsiveness in severe asthma populations to capture cough-related morbidity.

\section{Abbreviations}

A\&E: Accident and emergency; ACQ-6: Six-point juniper asthma control score; AQLQ: Asthma quality of life questionnaire; ATS: American thoracic society; BTS: British thoracic society; COPD: Chronic obstructive pulmonary disease; ERS: European respiratory society; FEV $_{1}$ : Forced expiratory volume in one second; FVC: Forced vital capacity; GINA: Global initiative for asthma; GLI: Global lung function initiative; GP: General practice; ICS/LABA: Inhaled corticosteroids/long acting beta-2 agonist combination therapy;

LCQ: Leicester cough questionnaire; MCID: Minimal clinically important difference; SD: Standard deviation

\section{Acknowledgements}

No applicable

\section{Funding}

This study was supported by the National Institute for Health Research Leicester Respiratory Biomedical Research Unit. The views expressed are those of the author(s) and not necessarily those of the NHS, the NIHR or the Department of Health.

\section{Availability of data and materials}

The datasets during and/or analysed during the current study available from the corresponding author on reasonable request.

\section{Authors' contributions}

SN analysed the data and wrote the manuscript. RCF generated data from the research database. PB reviewed the study manuscript and provided a scientific critique of the data. LM reviewed the study manuscript and provided a scientific critique of the data. SS conceived and designed the study, analysed the data and critically reviewed the manuscript. All authors read and approved the final manuscript.

\section{Competing interests}

PB has received grants from NIHR. SS has received grants and consultancy from Chiesi, NAPP pharmaceuticals, European Union FP7 Scheme: AirPROM-FP7, NHR, Sir Jules Thorne Trust, Astra-Zeneca, Glaxo Smith Kline and Boehringer Ingelheim and has received lecture fees from European Respiratory Society and Chiesi. SN, RCF and LM have no conflicts of interest to declare.

Consent for publication

No applicable.

\section{Ethics approval and consent to participate}

The use of data extracted from the local database for the purposes of research was approved by the Leicestershire, Northamptonshire, \& Rutland Research Ethics Committee (REC Reference.13/EM/0287) and all patients gave their written informed consent. 


\section{Author details}

Department of Infection, Immunity and Inflammation, NIHR Respiratory Biomedical Research Unit, Glenfield Hospital, University of Leicester, Leicester, UK. ${ }^{2}$ Centre for Infection \& Immunity, School of Medicine, Dentistry and Biomedical Sciences, Queen's University Belfast, Belfast, Ireland.

Received: 6 August 2016 Accepted: 9 February 2017

Published online: 04 March 2017

\section{References}

1. British Thoracic society/Scottish Intercollegiate Guidelines Network. British Guideline on the Managament of Asthma. 2016. Available at: https://www brit-thoracic.org.uk/document-library/clinical-information/asthma/btssignasthmaguideline-2016/.

2. O’Neill S, Sweeney J, Patterson CC, Menzies-Gow A, Niven R, Mansur AH, et al. The cost of treating severe refractory asthma in the UK: an economic analysis from the British Thoracic Society Difficult Asthma Registry. Thorax. 2015;70(4):376-8.

3. Sweeney J, Patterson CC, Menzies-Gow A, Niven RM, Mansur AH, Bucknall C, et al. Comorbidity in severe asthma requiring systemic corticosteroid therapy: cross-sectional data from the Optimum Patient Care Research Database and the British Thoracic Difficult Asthma Registry. Thorax. 2016;71(4):339-46.

4. Chung KF, Bel EH, Wenzel SE. Difficult-to-Treat Severe Asthma. European Respiratory Society Monographs ed. European Respiratory Society. 2011;51:1-15

5. Brignall K, Jayaraman B, Birring SS. Quality of life and psychosocial aspects of cough. Lung. 2008;186 Suppl 1:S55-8.

6. Morice AH, Millqvist E, Belvisi MG, Bieksiene K, Birring SS, Chung KF, et al. Expert opinion on the cough hypersensitivity syndrome in respiratory medicine. Eur Respir J. 2014;44(5):1132-48.

7. Birring SS, Fleming T, Matos S, Raj AA, Evans DH, Pavord ID. The Leicester Cough Monitor: preliminary validation of an automated cough detection system in chronic cough. Eur Respir J. 2008;31(5):1013-8.

8. Birring SS, Parker D, Brightling CE, Bradding P, Wardlaw AJ, Pavord ID. Induced sputum inflammatory mediator concentrations in chronic cough. Am J Respir Crit Care Med. 2004;169(1):15-9.

9. Lee KK, Matos S, Evans DH, White P, Pavord ID, Birring SS. A longitudinal assessment of acute cough. Am J Respir Crit Care Med. 2013;187(9):991-7.

10. Birring SS, Prudon B, Carr AJ, Singh SJ, Morgan MD, Pavord ID. Development of a symptom specific health status measure for patients with chronic cough: Leicester Cough Questionnaire (LCQ). Thorax. 2003;58(4):339-43.

11. Shirai T, Mikamo M, Tsuchiya T, Shishido Y, Akita T, Morita S, et al. Realworld effect of gastroesophageal reflux disease on cough-related quality of life and disease status in asthma and COPD. Allergol Int. 2015;64(1):79-83.

12. Berkhof FF, Boom LN, ten Hertog NE, Uil SM, Kerstjens HA, van den Berg JW. The validity and precision of the Leicester Cough Questionnaire in COPD patients with chronic cough. Health Qual Life Outcomes. 2012;10:4.

13. Chung KF, Wenzel SE, Brozek JL, Bush A, Castro M, Sterk PJ, et al. International ERS/ATS guidelines on definition, evaluation and treatment of severe asthma. Eur Respir J. 2014:43(2):343-73.

14. Raj AA, Pavord DI, Birring SS. Clinical cough IV:what is the minimal important difference for the Leicester Cough Questionnaire? Handb Exp Pharmacol. 2009;(187):311-20.

15. Heaney LG, Brightling CE, Menzies-Gow A, Stevenson M, Niven RM. British Thoracic Society Difficult Asthma Network. Refractory asthma in the UK: cross-sectional findings from a UK multicentre registry. Thorax. 2010;65(9):787-94.

16. Decalmer SC, Webster D, Kelsall AA, McGuinness K, Woodcock AA, Smith JA Chronic cough: how do cough reflex sensitivity and subjective assessments correlate with objective cough counts during ambulatory monitoring? Thorax. 2007;62(4):329-34.

17. Scholand MB, Wolff R, Crossno PF, Sundar K, Winegar M, Whipple $S$, et al. Severity of cough in idiopathic pulmonary fibrosis is associated with MUC5 B genotype. Cough. 2014;10:3. eCollection 2014.

18. Kastelik JA, Thompson RH, Aziz I, Ojoo JC, Redington AE, Morice AH. Sex-related differences in cough reflex sensitivity in patients with chronic cough. Am J Respir Crit Care Med. 2002;166(7):961-4.
19. Gupta S, Siddiqui S, Haldar P, Raj JV, Entwisle JJ, Wardlaw AJ, et al. Qualitative analysis of high-resolution CT scans in severe asthma. Chest. 2009;136(6):1521-8.

20. Gibson PG, Fujimura M, Niimi A. Eosinophilic bronchitis: clinical manifestations and implications for treatment. Thorax. 2002;57(2):178-82.

21. Costello RW, Schofield BH, Kephart GM, Gleich GJ, Jacoby DB, Fryer AD. Localization of eosinophils to airway nerves and effect on neuronal M2 muscarinic receptor function. Am J Physiol. 1997;273(1 Pt 1):L93-103.

22. Abdulqawi R, Dockry R, Holt K, Layton G, McCarthy BG, Ford AP, et al. P2X3 receptor antagonist (AF-219) in refractory chronic cough: a randomised, double-blind, placebo-controlled phase 2 study. Lancet. 2015;385(9974):1198-205.

\section{Submit your next manuscript to BioMed Central and we will help you at every step:}

- We accept pre-submission inquiries

- Our selector tool helps you to find the most relevant journal

- We provide round the clock customer support

- Convenient online submission

- Thorough peer review

- Inclusion in PubMed and all major indexing services

- Maximum visibility for your research

Submit your manuscript at www.biomedcentral.com/submit 